\title{
Experimental Signatures of Low Energy Gauge-Mediated Supersymmetry Breaking
}

\author{
Savas Dimopoulos, ${ }^{1,2}$ Michael Dine, ${ }^{3}$ Stuart Raby, ${ }^{4}$ and Scott Thomas ${ }^{5}$ \\ ${ }^{1}$ Physics Department, Stanford University, Stanford, California 94309 \\ ${ }^{2}$ Theoretical Physics Division, CH-1211, Geneva 23, Switzerland \\ ${ }^{3}$ Santa Cruz Institute for Particle Physics, University of California, Santa Cruz, California 95064 \\ ${ }^{4}$ Physics Department, The Ohio State University, Columbus, Ohio 43210 \\ ${ }^{5}$ Stanford Linear Accelerator Center, Stanford, California 94309
}

(Received 31 January 1996)

\begin{abstract}
The experimental signatures for low energy gauge-mediated supersymmetry breaking are distinctive since the gravitino is naturally the lightest supersymmetric particle. The next lightest supersymmetric particle (NLSP) can be a gaugino, Higgsino, or right handed slepton. For a significant range of parameters, decay of the NLSP to its partner plus the gravitino can be measured as a displaced vertex or kink in a charged particle track. In the case that the NLSP is mostly gaugino, we identify the discovery modes as $e^{+} e^{-} \rightarrow \gamma \gamma+\mathbb{E}$, and $p \bar{p} \rightarrow l^{+} l^{-} \gamma \gamma+\mathbb{E}_{T}$. If the NLSP is a right-handed slepton, the discovery modes are $e^{+} e^{-} \rightarrow l^{+} l^{-}+\not \mathbb{E}$ and $p \bar{p} \rightarrow l^{+} l^{-}+\mathbb{E}_{T}$. A NLSP which is mostly Higgsino is also considered. [S0031-9007(96)00048-8]
\end{abstract}

PACS numbers: 14.80.Ly, 11.30.Qc, 12.60.Jv

Low energy supersymmetry is widely viewed as a plausible solution of the hierarchy problem. If nature is supersymmetric, it is important to understand how supersymmetry is broken. It is usually assumed that supersymmetry breaking is communicated to ordinary fields and their superpartners by supergravity. The breaking scale is then necessarily of order $10^{11} \mathrm{GeV}$. An alternative possibility, which has been less thoroughly explored, is that supersymmetry is broken at some lower energy scale, and that the ordinary gauge interactions act as the messengers of supersymmetry breaking [1-3]. In this case, the scale of supersymmetry breaking can be as low as tens of $\mathrm{TeV}[1-3]$.

Independent of source and messenger, supersymmetry breaking is represented among ordinary fields (the visible sector) by soft supersymmetry breaking terms [4]. The most general soft-breaking Lagrangian is described by 105 parameters beyond those of the minimal standard model [5]. There are a number of constraints which these parameters must satisfy, coming from direct experimental searches for superpartners, electric dipole moments, and the lack of flavor changing neutral currents. Most model builders simply postulate a high degree of degeneracy among squarks and sleptons at a high energy scale to deal with this problem [4]. In certain classes of superstring theories, there are weak hints for such a universality $[6,7]$. Alternatively, the various experimental constraints might be satisfied as a result of flavor symmetries or by other means [8-10]. With gauge-mediated supersymmetry breaking the entire soft-breaking Lagrangian can be calculated in terms of a small number of parameters. In addition, the regularities required to avoid flavor changing neutral currents are automatically obtained since the ordinary gauge interactions do not distinguish generations. For these reasons, we believe the gauge-mediated possibility should be taken seriously.
In this Letter, we discuss some striking and distinctive signatures of low energy gauge-mediated supersymmetry breaking. The first is the spectrum of superpartner masses. These masses are functions of the gauge quantum numbers, and are roughly in the ratio of the appropriate gauge couplings squared. In the simplest models, definite relations exist among these masses. As a result, the lightest standard model superpartner is almost inevitably either a neutralino or a right-handed slepton. The second important signature arises from the fact that the lightest supersymmetric particle (LSP) is the gravitino. The lightest standard model superpartner is then the next to lightest supersymmetric particle (NLSP). Assuming that $R$ parity is conserved, the principle decay of the NLSP is then to its partner plus a gravitino. The longitudinal component of the gravitinothe Goldstino-couples to matter with strength proportional to $F^{-1}$, where $F$ is the scale of supersymmetry breaking. For a plausible range of $F$, the decay length can be hundreds of $\mu \mathrm{m}$ to meters. The decays can therefore take place within a detector. This leads to signatures for supersymmetry which are distinct from the conventional minimal supersymmetric standard model (MSSM), and with potentially dramatic displaced vertices.

Superpartner spectrum. - In gauge-mediated models, supersymmetry is broken in a messenger sector which transforms under the standard model gauge group. The matter fields in this sector are generally referred to as messenger quarks and leptons. Supersymmetry breaking is transmitted to the visible sector by ordinary gauge interactions. To preserve the successful supersymmetric prediction of the low energy $\sin ^{2} \theta_{W}$, it is sufficient that the messengers form a grand unified theory (GUT) representation. In the simplest versions, the messenger fields are weakly coupled and possess the quantum numbers of a single $\mathbf{5}+\overline{\mathbf{5}}$ of SU(5), i.e., there are triplets, $q$ and $\bar{q}$, and doublets, $\ell$ and $\bar{\ell}$. They couple to a single 
gauge singlet field, $S$, through a superpotential $W=$ $\lambda_{1} S_{q \bar{q}}+\lambda_{2} S \ell \bar{\ell}$. The field $S$ has nonzero expectation values for both scalar and auxiliary components, $S$ and $F_{S}$. Integrating out the messenger sector then gives rise to gaugino masses at one loop. For $F_{S} \ll S$, these masses are given by [3]

$$
m_{\lambda_{i}}=c_{i} N \frac{\alpha_{i}}{4 \pi} \Lambda,
$$

where $c_{1}=\frac{5}{3}, c_{2}=c_{3}=1, \Lambda=F_{S} / S$, and for a more general messenger sector $N$ is the equivalent number of $\mathrm{SU}(5) \mathbf{5}+\overline{\mathbf{5}}$ representations. The scalar masses squared arise at two loops [3],

$\tilde{m}^{2}=2 \Lambda^{2} N\left[C_{3}\left(\frac{\alpha_{3}}{4 \pi}\right)^{2}+C_{2}\left(\frac{\alpha_{2}}{4 \pi}\right)^{2}+\frac{5}{3}\left(\frac{Y}{2}\right)^{2}\left(\frac{\alpha_{1}}{4 \pi}\right)^{2}\right]$,

where $C_{3}=\frac{4}{3}$ for color triplets and zero for singlets, $C_{2}=\frac{3}{4}$ for weak doublets and zero for singlets, and $Y$ is the ordinary hypercharge normalized as $Q=T_{3}+\frac{1}{2} Y$. It should be stressed that $F_{S}$ is not necessarily the intrinsic supersymmetry breaking scale, $F$, since the gauge singlet field may not be coupled directly to the supersymmetry breaking sector. For example, in the model of Ref. [3], $F \gg F_{S}$. However, it is also perfectly possible that $F \sim$ $F_{S}$ [11]. While $F_{S}$ determines the superpartner masses, it is $F$ which determines the Goldstino coupling discussed below.

These expressions for the masses possess a number of noteworthy features. There is a hierarchy of masses, with colored particles being the most massive, and $\mathrm{SU}(3) \times$ $\mathrm{SU}(2)$ singlet particles the lightest. The gaugino masses are in the ratio $7: 2: 1$, just as for supersymmetry breaking with universal gaugino masses at a high scale. For $N=1$ the squark, left-handed slepton, right-handed slepton, and $B$-ino (partner of the hypercharge gauge boson) masses are in the ratio $11.6: 2.5: 1.1: 1$. In this case the $B$-ino is the natural candidate for the NLSP. The gaugino masses grow as $N$, while the scalar masses grow as $\sqrt{\mathrm{N}}$. For $N=2$ the above masses are in the ratio $10.6: 2.3: 1: 1.3$. In this case the right-handed slepton is the candidate for the NLSP.

In more general models the above relations among the masses can be modified. For example, both (1) and (2) are corrected at $\mathcal{O}\left(F / \lambda S^{2}\right)$. Additional modifications can arise with several gauge singlet fields coupling to $q \bar{q}$ and $\ell \bar{\ell}$. In the model with one singlet, the couplings $\lambda_{1}$ and $\lambda_{2}$ cancel out in the expressions for the masses, but this is not true of the more general case. As a result, both the ratios of the squark and slepton masses and the ratio of these masses to gaugino masses are modified. More generally, scalar masses require only supersymmetry breaking, while gaugino masses also require that $\mathrm{U}(1)_{R}$ be broken to, at most, $R$ parity. In principle, $\mathrm{U}(1)_{R}$ could effectively be broken at a lower scale than supersymmetry, leading to gauginos which are much lighter than the scalars.

Perhaps a more interesting possibility is that the messenger sector is strongly coupled. Gaugino masses can then arise directly from nonperturbative dynamics in the messenger sector, $m_{\lambda} \sim \alpha \Lambda$. The scalar masses require one perturbative gauge loop, $\tilde{m}^{2} \sim \alpha(\alpha / 4 \pi) \Lambda^{2}$. So, in this case, the gauginos are much heavier than the scalars, and the natural candidate for the NLSP is the right-handed slepton. All of the possibilities given above for the messenger sector have in common the feature that masses for standard model superpartners go roughly as gauge couplings squared, although the relation of scalar to gaugino masses is model dependent.

The dimensionful terms which must arise in the Higgs sector $W=\mu H_{1} H_{2}$, and $V=m_{12}^{2} H_{1} H_{2}+$ H.c., do not follow directly from the ansatz of gauge-mediated supersymmetry breaking, and are model dependent. This is because these terms require that the Peccei-Quinn symmetry between $H_{1}$ and $H_{2}$ be broken by nongauge interactions. Specific models with additional singlets and vector quarks have been constructed in which $\mu$ and $m_{12}^{2}$ do arise with reasonable magnitude [3]. Because the properties of the Higgs sector are not generic, we leave open the possibility that the lightest electroweak neutralino is a general mixture of gaugino and Higgsino.

Phenomenology. - Perhaps the most dramatic consequence of low energy gauge-mediated supersymmetry breaking is that the gravitino is the LSP. In the global limit the Goldstone fermion, or Goldstino, of supersymmetry breaking is massless. In local supersymmetry, the Goldstino becomes the longitudinal component of the gravitino, giving a gravitino mass (assuming the cosmological constant vanishes) of

$$
m_{G}=\frac{F}{\sqrt{3} M_{p}} \simeq 2.5\left(\frac{F}{(100 \mathrm{TeV})^{2}}\right) \mathrm{eV},
$$

where $F$ is the supersymmetry breaking scale. The lightest standard model supersymmetric particle is then the NLSP, and can decay to its partner and the gravitino. The lowest order coupling of the Goldstino is fixed by the supersymmetric Goldberger-Treiman low energy theorem to be given by [12]

$$
\mathcal{L}=-\frac{1}{F} j^{\alpha \mu} \partial_{\mu} G_{\alpha}+\text { H.c. }
$$

where $j^{\alpha \mu}$ is the supercurrent and $G_{\alpha}$ is the spin $\frac{1}{2}$ longitudinal Goldstino component of the gravitino. The decay to the Goldstino component is then suppressed only by $F$ rather than $M_{p}$. In the case that the NLSP is mostly $B$-ino, $\tilde{B}$, the coupling (4) leads to a transition magnetic dipole moment between the NLSP and gravitino, $\cos \theta_{W}\left(m_{\tilde{B}} / 2 \sqrt{2} F\right) \tilde{B} \bar{\sigma}^{\mu} \sigma^{\nu} G F_{\mu \nu}+$ H.c., giving rise to a decay rate

$$
\Gamma(\tilde{B} \longrightarrow G+\gamma)=\frac{\cos ^{2} \theta_{W} m_{\tilde{B}}^{5}}{16 \pi F^{2}} .
$$

This translates to a decay length

$$
c \tau \simeq 130\left(\frac{100 \mathrm{GeV}}{m_{\tilde{B}}}\right)^{5}\left(\frac{\sqrt{F}}{100 \mathrm{TeV}}\right)^{4} \mu \mathrm{m} .
$$

So there is a range of $F$ and $m$ for which the decay occurs within the detector, with the gravitino carrying 
off missing energy. For $m_{\tilde{B}}>m_{Z}^{0}$ there is also a nonnegligible branching fraction $\tilde{B} \rightarrow G+Z^{0}[B(\tilde{B} \rightarrow G+$ $\left.Z^{0}\right) \rightarrow \sin ^{2} \theta_{W}$ for $m_{\tilde{B}} \gg m_{Z}$ ]. In the case that the NLSP is a right-handed slepton, it can decay by $\tilde{l}_{R} \rightarrow G+$ $l_{R}$ with a decay length similar to (6). If the NLSP is mostly Higgsino, it can decay by $\tilde{H}^{0} \rightarrow G+h^{0}$ if $m_{h^{0}}<m_{\tilde{H}}$, where $h^{0}$ is the lightest Higgs boson. For $m_{h^{0}}>m_{\tilde{H}}$ decay $\tilde{H}^{0} \rightarrow G+b \bar{b}$ is possible; however, for reasonable values of the parameters the NLSP decays predominantly to $G+\gamma$ through its gaugino components.

Decay of the lightest standard model supersymmetric particle to its partner plus the gravitino within the detector gives signatures which are distinct from the conventional MSSM. Let us focus on the discovery modes at $e^{+} e^{-}$and hadron colliders. Consider first the case in which the NLSP is mostly $B$-ino. At $e^{+} e^{-}$colliders, $e^{+} e^{-} \rightarrow \tilde{B} \tilde{B} \rightarrow \gamma \gamma+\not{E}$ is dominated by $t$ - and $u$-channel right-handed selectron exchange. The production cross section for this process can be significant. For example, with $\sqrt{s}=2.2 m_{\tilde{B}}$, and assuming the spectrum resulting from the simple model with $N=1$ given in the previous section, $\sigma\left(e^{+} e^{-} \rightarrow \tilde{B} \tilde{B}\right) \simeq 0.87 R$ where $R=4 \pi \alpha^{2} / 3 s$ is the $e^{+} e^{-} \rightarrow \mu^{+} \mu^{-}$cross section. In many models, since the $B$-ino and slepton masses are related, the total cross section is related to the $B$-ino mass. This process should show significant polarization dependence since $\tilde{e}_{R}$ is lighter than $\tilde{e}_{L}$, and the hypercharge of $\tilde{e}_{R}$ is twice that of $\tilde{e}_{L}$. For the parameters given above, $\sigma\left(e^{+} e_{L}^{-} \rightarrow \tilde{B} \tilde{B}\right) / \sigma\left(e^{+} e_{R}^{-} \rightarrow \tilde{B} \tilde{B}\right) \simeq 0.01$. The $B$-ino decay is isotropic in the rest frame, implying that the photons have a flat energy distribution in the laboratory frame. Cuts on the $\gamma \gamma$ invariant mass can easily eliminate the background from $e^{+} e^{-} \rightarrow \gamma \gamma Z^{0}$ with $Z^{0} \rightarrow \nu \bar{\nu}$.

The signature $\gamma \gamma+\not \mathbb{E}$ can also arise in the conventional MSSM in some regions of parameter space if the LSP is mostly Higgsino. In this case the NLSP is not much heavier than the LSP, is also mostly Higgsino, and has a significant branching ratio $\tilde{H}_{2} \rightarrow \tilde{H}_{1}+\gamma \cdot e^{+} e^{-} \rightarrow \tilde{H}_{2}^{0} \tilde{H}_{2}^{0}$ then gives rise to this mode. In the gauge-mediated case, however, since $\not{E}$ is carried by the essentially massless gravitinos, the photon energy is bounded by $\frac{1}{4} \sqrt{s}(1-\beta) \leq E_{\gamma} \leq \frac{1}{4} \sqrt{s}(1+\beta)$, where $\beta=$ $\sqrt{1-4 m_{\tilde{B}}^{2} / s}$ is the $B$-ino velocity. In the conventional case, since $\not E$ is carried by the massive LSP, the photon energy end points are smaller by a factor $\left(1-m_{\tilde{H}_{1}^{0}}^{2} / m_{\tilde{H}_{2}^{0}}^{2}\right)$, where $\beta$ in this case is the $\tilde{H}_{2}^{0}$ velocity. This allows the decay to a gravitino to be distinguished from decay to the LSP in the conventional MSSM. In addition, in this region of parameter space the lightest chargino is just slightly heavier, is also mostly Higgsino, and decays predominantly by $\tilde{H}^{ \pm} \rightarrow \tilde{H}^{0} W^{ \pm *}$. In the MSSM the additional signatures $e^{+} e^{-} \rightarrow \tilde{H}^{+} \tilde{H}^{-} \rightarrow 4 j+\not{E}, j j l+\not k$, and $l^{+} l^{\prime-}+\not \mathbb{E}$ are likely to be accessible at comparable $\sqrt{s}$. This is in contrast to the gauge-mediated case with a mostly $B$-ino NLSP.

As discussed in the previous section, with a weakly coupled messenger sector giving a NLSP which is mostly
$B$-ino, it is likely that the right-handed sleptons are not too much heavier than the NLSP. In this case, in addition to $B$-ino pair production, slepton pair production may be kinematically accessible. Cascade decay through the $B$ ino then gives rise to $e^{+} e^{-} \tilde{l}_{R}^{+} \tilde{l}_{R}^{-} \rightarrow l^{+} l^{-} \gamma \gamma+\not{E}$.

If the NLSP is a right-handed slepton, the discovery mode is $e^{+} e^{-} \rightarrow \tilde{l}_{R}^{+} \tilde{l}_{R}^{-} \rightarrow l^{+} l^{-}+\not E$. As for the decay to photons, the leptons have a flat energy distribution, with end points determined by $\sqrt{s}$ and $m_{l_{R}}$. The final states with $e, \mu$, and $\tau$ should appear with very nearly equal $m_{\tau_{R}}$. Cuts on $\not \mathbb{E}$ can easily eliminate the background $e^{+} e^{-} \rightarrow Z^{0} l^{+} l^{-}$with $Z^{0} \rightarrow \nu \bar{\nu}$. This signature can also arise in the conventional MSSM, where the missing energy is carried by the massive LSP. However, the lepton energy end points again distinguish this from an essentially massless gravitino. It is interesting to note that if $\sqrt{F}$ is much larger than a few $1000 \mathrm{TeV}$ the decay of $\tilde{l}_{R}$ takes place well outside the detector. The signature for supersymmetry is then massive charged particles, rather than the traditional missing energy.

If the NLSP is mostly Higgsino, and $m_{\tilde{H}}>m_{h^{0}}$, the discovery mode is $e^{+} e^{-} \rightarrow \tilde{H}^{0} \tilde{H}^{0} \rightarrow 4 b+\not{E}$, with, of course, two pairs of $b$ jets reconstructing the Higgs mass. In this part of parameter space the next heaviest neutralino and lightest chargino are mostly Higgsino, not much heavier than $\tilde{H}^{0}$, and have the same decay modes to $\tilde{H}^{0}$ as in the MSSM. The signatures $e^{+} e^{-} \rightarrow \tilde{H}^{+} \tilde{H}^{-} \rightarrow 4 b 4 j+$ $\not E, 4 b j j l+\not E$, and $4 b l^{+} l^{\prime-}+\not E$ should therefore also be accessible at comparable $\sqrt{s}$, with the additional jets and leptons fairly soft.

The discovery modes at hadron colliders can be somewhat different than for $e^{+} e^{-}$colliders. If the NLSP is very nearly purely $B$-ino, $p \bar{p} \rightarrow \tilde{B} \tilde{B} \rightarrow \gamma \gamma+\not_{T}$ proceeds predominantly through $t$ - and $u$-channel squark exchange, and is therefore highly suppressed because of the large squark masses. However, sleptons can be pair produced by the Drell-Yan process. Cascade decay through the $B$-ino then leads to $p \bar{p} \rightarrow \tilde{l}_{R}^{+} \tilde{l}_{R}^{-} \rightarrow l^{+} l^{-} \gamma \gamma+\mathscr{H}_{T}$. One such spectacular $e e \gamma \gamma$ event has in fact been observed at the Tevatron by the CDF collaboration (event 257646 in run 68739) [13]. The obvious background from $p \bar{p} \rightarrow$ $W W \gamma \gamma$ has a very small production rate, and would give rise to other decays modes which are not observed [14]. In contrast, the production cross section for $p \bar{p} \rightarrow \tilde{l}_{R}^{+} \tilde{l}_{R}^{-}$with $m_{\tilde{l}_{R}} \simeq 95 \mathrm{GeV}$ is roughly $10^{-2} \mathrm{pb}$ [15]. With $\sim 100 \mathrm{pb}^{-1}$ of integrated luminosity, the single observed event could be consistent with right-handed slepton pair production. The kinematics of this event favor a fairly light $B$-ino, implying that $e^{+} e^{-} \rightarrow \tilde{B} \tilde{B} \rightarrow \gamma \gamma+\not E^{*}$ is likely to be observed at LEPII.

If the sleptons are much heavier than the gauginos, and the NLSP is mostly $B$-ino, pair production of $W$-inos becomes the dominant production mechanism, $p \bar{p} \rightarrow$ $W^{*} \rightarrow \tilde{W}^{ \pm} \tilde{W}^{0}$. The dominant $W$-ino decay modes are $\tilde{W}^{ \pm} \rightarrow \tilde{B} W^{ \pm *}$ and $\tilde{W}^{0} \rightarrow \tilde{B} Z^{0 *}$ through mixing with the Higgsino states, and $\tilde{W}^{ \pm} \rightarrow \tilde{B} l \nu$ and $\tilde{W}^{0} \rightarrow \tilde{B} l^{+} l^{-}$ through off-shell sleptons. Cascade decays through 
the $B$-ino then lead to the signatures $p \bar{p} \rightarrow \tilde{W}^{ \pm} \tilde{W}^{0} \rightarrow$ $4 j \gamma \gamma+\mathbb{E}_{T}$, jjl $\gamma \gamma+\mathbb{E}_{T}$, and $l^{+} l^{-} l^{\prime} \gamma \gamma+\mathbb{E}_{T}$. The last one is similar to the standard trilepton signature of chargino pair production [16]. Here, the additional hard photons significantly reduce the background. If the NLSP is mostly Higgsino or a right-handed slepton, the signatures at hadron colliders are similar to those at $e^{+} e^{-}$.

By far the most dramatic signature of low energy supersymmetry breaking is the possibility of measuring directly the decay of the NLSP to its partner plus the gravitino. If the NLSP is a neutralino, this appears as a displaced vertex, while for a slepton NLSP it appears as a kink in a charged particle track. Measurement of the decay distribution would allow a direct determination of the supersymmetry breaking scale. For the decay of right-handed sleptons to leptons, or the decay of Higgsinos to the lightest Higgs boson, tracking of the resulting charged particles in a silicon vertex detector and central tracking region would allow measurements of $\mathrm{c} \tau$ between roughly $100 \mu \mathrm{m}$ and $10 \mathrm{~m}$. In the case of decay to a photon, the tracking ability for the displaced vertex is generally not good. However, if such a signal were established experimentally, detectors could be optimized to convert photons within the tracking region. So, depending on the specific decay modes of the NLSP, displaced vertices for $\sqrt{F}$ between roughly 100 and $1000 \mathrm{TeV}$ could be accessible to collider experiments.

This range of experimentally accessible $\sqrt{F}$ is, in fact, consistent with astrophysical and cosmological considerations. Unless there is an inflation with low reheat temperature, avoiding overclosure of the Universe from relic gravitinos requires $\sqrt{F} \lesssim 2 \times 10^{3} \mathrm{TeV}$. In many theories a potentially dangerous $R$ axion arises in the supersymmetry breaking sector [17]. For $\sqrt{F}$ above a few TeV, $R$-violating interactions suppressed by a single power of the Planck scale make the $R$ axion too heavy to be produced during helium ignition in red giants [18]. In addition, it is either trapped or too heavy to deplete the neutrino pulse from SN1987A. Finally, for weakly coupled models with a single additional scale, such as the simple example presented previously with $F_{S} \sim F$, electroweak scale superpartners are obtained for $\sqrt{F} \sim 100 \mathrm{TeV}$.

A final possible consequence of these theories is that scalar moduli with Planck suppressed couplings to matter obtain masses of order or smaller than the gravitino mass as the result of supersymmetry breaking. These fields can mediate coherent forces in the submillimeter range, which has not been explored experimentally. New techniques employing small cryogenic mechanical oscillators [19] or atomic beams [20] may allow the detection of such short range gravitational strength forces.

Low energy gauge-mediated supersymmetry breaking clearly makes distinct and dramatic predictions for future experiments. The new particle spectrum is predicted in terms of a small number of parameters. For a quite plausible range of these parameters, it predicts signatures distinctly different than those of the conventional MSSM.
Most dramatic of these is the possibility of measuring displaced vertices or kinks in charged particle tracks from decays to the gravitino.

We would like to thank G. Anderson, R. Barbieri, G. Giudice, H. Haber, L. Hall, M. Peskin, A. Pomarol, and J. Wells for valuable discussions. This work was supported by the Department of Energy (M.D.), by the Department of Energy under Contract DOE-ER-01545646 (S.R.), and under Contract DE-AC03-76SF00515 (S. T.).

[1] M. Dine, W. Fischler, and M. Srednicki, Nucl. Phys. B189, 575 (1981); S. Dimopoulos and S. Raby, Nucl. Phys. B192, 353 (1981); M. Dine and W. Fischler, Phys. Lett. 110B, 227 (1982); M. Dine and M. Srednicki, Nucl. Phys. B202, 238 (1982); L. Alvarez-Gaumé, M. Claudson, and M. Wise, Nucl. Phys. B207, 96 (1982); C. Nappi and B. Ovrut, Phys. Lett. 113B, 175 (1982).

[2] M. Dine and W. Fischler, Nucl. Phys. B204, 346 (1982); S. Dimopoulos and S. Raby, Nucl. Phys. B219, 479 (1983).

[3] M. Dine, A. E. Nelson, and Y. Shirman, Phys. Rev. D 51, 1362 (1995); M. Dine, A.E. Nelson, Y. Nir, and Y. Shirman, Phys. Rev. D 53, 2658 (1996).

[4] S. Dimopoulos and H. Georgi, Nucl. Phys. B193, 150 (1981).

[5] S. Dimopoulos and H. Georgi, Nucl. Phys. B452, 496 (1995).

[6] I. E. Ibanez and D. Lust, Nucl. Phys. B382, 305 (1992).

[7] V. Kaplunovsky and J. Louis, Phys. Lett. B 306, 269 (1993).

[8] M. Leurer, Y. Nir, and N. Seiberg, Phys. Lett. B 309, 337 (1993).

[9] M. Dine, A. Kagan, and R. Leigh, Phys. Rev. D 48, 4269 (1993).

[10] D. B. Kaplan and M. Schmalz, Phys. Rev. D 49, 3741 (1994).

[11] K. Intriligator and S. Thomas, Report No. SLAC-PUB-957041, hep-th/9603158 (to be published).

[12] P. Fayet, Phys. Lett. B 84, 416 (1979); Phys. Rep. 105, 21 (1984).

[13] S. Park representing the CDF Collaboration, in 10th Topical Workshop on Proton-Antiproton Collider Physics, Illinois, 1995, edited by R. Raja and J. Yoh, AIP Conf. Proc. No. 357 (AIP, New York, 1996).

[14] R. Culbertson, H. Frisch, and D. Toback, CDF Collaboration, Report No. CDF-3456, 1995.

[15] H. Baer, C.-h. Chen, F. Paige, and X. Tata, Phys. Rev. D 49, 3283 (1994).

[16] H. Baer, K. Hagiwara, and X. Tata, Phys. Rev. D 35, 1598 (1987); P. Nath and R. Arnowitt, Mod. Phys. Lett. A 2, 331 (1987).

[17] A. Nelson and N. Seiberg, Nucl. Phys. B416, 46 (1994); J. Bagger, E. Poppitz, and L. Randall, Nucl. Phys. B426, 3 (1994).

[18] G. Raffelt, Phys. Rep. 198, 1 (1990).

[19] P. Michelson, D. Osheroff, and J. Price, in Proceedings of the International Symposium on Experimental Gravitational Physics, edited by P. Michelson, H. En-ke, and G. Pizzella (World Scientific, Singapore, 1987), p. 436.

[20] M. Kasevitch (private communication). 